\title{
Plant secondary metabolites: antiparasitic effects and their role in ruminant production systems
}

\author{
Spiridoula Athanasiadou* and Ilias Kyriazakis \\ Animal Nutrition and Health Department, Scottish Agricultural College, West Mains Road, Edinburgh EH9 3JG, UK
}

\begin{abstract}
The purported antiparasitic properties of plant secondary metabolites (PSM) have been the cause of controversy amongst the scientific community. Despite long-standing knowledge of the prophylactic and therapeutic properties of PSM-rich extracts, which comes mainly from ethnoveterinary sources, the scientific evidence of the antiparasitic effects of PSM is inconsistent. In the first part of the present paper the causes of this controversy are addressed, and the evidence available on the antiparasitic effects of PSM is critically examined. The focus is on examples of the antiparasitic activity of PSM against helminth nematodes. The conclusion is that PSM can have antiparasitic properties, which depend on their structure, level of ingestion and availability within the gastrointestinal tract of the animal. The second part is an appraisal of the potential role of PSM for parasite control in ruminant production systems. Despite their antiparasitic properties, PSM consumption does not necessarily have positive consequences in parasitised herbivores, as excessive consumption of PSM can adversely affect herbivore fitness and survival, through their anti-nutritional properties. For this reason it is suggested that the antiparasitic properties of PSM should be assessed at the same time as their anti-nutritional effects. The same measure, e.g. the performance of parasitised hosts, should be used when assessing these properties. The assessment of the costs and benefits suggests that parasitised herbivores can benefit from the long-term consumption of PSM only if the antiparasitic benefits outweigh the anti-nutritional costs of PSM. In addition, it is proposed that parasitised animals might be able to benefit from PSM consumption even if their performance is impaired, as long as the latter is a short-term compromise that leads to long-term benefits.
\end{abstract}

Bioactive plants: Gastrointestinal parasites: Helminths: Plant secondary metabolites

Gastrointestinal parasitism has been classified as a major health and welfare problem for ruminants. Parasitism, especially by helminth parasites, impairs health by causing inappetance, diarrhoea, anaemia and, in severe cases, death (Table 1). In addition to compromising health and welfare, parasitism impairs productivity and results in poor growth and reproduction, e.g. the reduction in body-weight gain by $\leq 70 \%$ within a few weeks of infection (Coop et al. 1982). Since the 1960s, when the first anthelmintic drugs for gastrointestinal parasite control became available, chemoprophylaxis has been the main method of control. However, the emergence of resistance to anthelmintic drugs, which is now a worldwide phenomenon (Jackson \& Coop, 2000), together with the increased awareness of consumers about drug residues that potentially enter the food chain, has stimulated investigation into alternative approaches to the control of gastrointestinal parasitism in ruminants. Alternative approaches to the use of anthelmintics that are currently under investigation include: biological control of nematodes by using nematophagous fungi (Larsen, 1999); breeding livestock for nematode resistance (Gray, 1997); nutrient supplementation of parasitised herbivores (Houdijk et al. 2001); development of vaccines against helminths (Smith, 1999); the consumption of bioactive forages (Coop \& Kyriazakis, 2001). The purpose of the present paper is to consider further the use of bioactive forages to control parasitism in ruminant production systems. Bioactive forages are those that contain compounds that are active against pathogens; in the case of parasitism, the pathogens are the parasites. Although in many cases the active compounds of the bioactive forages are still unidentified, in those cases 
Table 1. Epidemiologically-important gastrointestinal nematodes of sheep, goats and cattle, their site of infection and main effect on the parasitised host (adapted from Houdijk \& Athanasiadou, 2003)

\begin{tabular}{llll}
\hline & Site of infection & \multicolumn{1}{c}{ Nematodes } & Consequences of parasitism \\
\hline Sheep, goats & Abomasum & Haemonchus contortus & Anaemia \\
& Seladorsagia circumcincta & Reduced food intake \\
& Small intestine & Nematodirus battus & Dehydration \\
& & Trichostrongylus colubriformis & Reduced food efficiency \\
Cattle & Trichostrongylus vitrinus & Reduced food efficiency \\
& Abomasum & Haemonchus placei & Anaemia \\
& Small intestine & Ostertagia ostertagi & Reduced food intake \\
& & Cooperia oncophora & Reduced food efficiency \\
\hline
\end{tabular}

where they have been identified they are plant secondary metabolites (PSM).

As the term implies, PSM are plant compounds that are not strictly essential to the main functions of the plants, such as their growth and reproduction. They have been associated, however, with plant defence mechanisms against herbivory (Harborne, 1999; Karban et al. 1999). Plant parts or extracts rich in PSM have been used to combat parasitism for many centuries, and in many parts of the world such products are still used for this purpose (International Institute of Rural Reconstruction, 1994). There is evidence of the antiparasitic effects of PSM against helminths, such as nematodes, and also against protozoa and external parasites (International Institute of Rural Reconstruction, 1994). Despite the long-standing, but mainly anecdotal, evidence of the antiparasitic effects of PSM-rich plants, there is controversy amongst parasitologists, nutritionists and animal scientists about their efficacy. The main reason for this controversy is that there is no generally accepted evidence on the antiparasitic effects of PSM. Thus, before assessing the potential role of PSM for parasite control, it is first necessary to critically evaluate the evidence for the antiparasitic properties of PSM. It will be shown that PSM can exert antiparasitic effects, the extent of which depends on their chemical structure, the level of ingestion and the site of parasitism.

In the second part of the paper it will be argued that even though PSM may have antiparasitic properties, these properties do not necessarily translate into beneficial consequences for parasitised ruminants. The reason is that the majority of PSM also have anti-nutritional properties. While most PSM deter herbivores from ingesting plants, there are other PSM that act as toxins and cause temporary or permanent physiological changes in herbivores (Bernays et al. 1989; Harborne, 1999). In order to determine the consequences of PSM consumption, it is proposed that the antiparasitic benefits and the anti-nutritional costs of PSM consumption should be considered together. It will be suggested that future research should include this simultaneous evaluation of the positive and negative effects of PSM; this approach is currently rare. In the final part of the paper the research that is required will be further explored before PSM consumption for parasite control is incorporated into ruminant production systems.

\section{Antiparasitic properties of plant secondary metabolites: fact or fiction?}

In ethnoveterinary medicine, which draws inspiration from traditional practice, there seems to be a plant or plant extract suitable for treating each parasitic disease (International Institute of Rural Reconstruction, 1994). For example, seeds of garlic (Allium sativum), onion (Allium сера) and mint (Mentha spp.) have been used to treat animals that suffer from gastrointestinal parasitism, whereas extracts of the tobacco plant (Nicotiana tabacum) have been used to treat the skin of livestock afflicted with the external parasite that causes scabies (Guarrera, 1999). Leaves, dried flowers and oil from Chenopodium ambrosioides, a shrub that originated from Central America and has been distributed around the world, have all been used as anthelmintics since the early 1900s (Guarrera, 1999). Table 2 shows examples of plants that have been reported by ethnoveterinary sources to have antiparasitic properties. Although ethnoveterinary reports are the main source for the widely-held perception of the antiparasitic properties of plants, they offer little quantitative evidence of such antiparasitic effects, which would be necessary for the incorporation of such plants in parasite control strategies. The evidence from quantitative studies, which may be either in vitro or in vivo, and from ethnoveterinary reports is sometimes contradictory (Githiori et al. 2002; Ketzis et al. 2002). The inconsistency has led to doubt about the antiparasitic effects of PSM. Here, the grounds for this contradiction will be explored. The focus will be on the two issues believed to be the main causes of the scientific confusion: first the apparent conflict between the evidence coming from in vitro and in vivo studies; second the inconsistent results of in vivo studies.

\section{Conflicting evidence between in vitro and in vivo studies}

Scientific evidence supporting the antiparasitic properties of PSM comes mainly from in vitro studies. The main advantage of using in vitro assays in this context is that purified compounds can be tested; other advantages include their low cost and rapid turnover. Testing purified compounds allows their activity to be reliably quantified without the interference of other plant components or nutrients. In vitro assays allow assessment of the effects of 
Table 2. Examples of plants that have been reported by ethnoveterinary sources to be effective against gastrointestinal parasites

\begin{tabular}{|c|c|c|c|c|}
\hline Plant or forage & $\begin{array}{l}\text { Parasite } \\
\text { class }\end{array}$ & $\begin{array}{l}\text { Continent of } \\
\text { origin }\end{array}$ & $\begin{array}{l}\text { Suspected active } \\
\text { compound }\end{array}$ & Reference \\
\hline Hedera helix & Cestode & Europe & Saponins & Julien et al. (1985) \\
\hline Punica granatum & Helminth* & Europe & Polyphenols & Guarrera (1999) \\
\hline Artemisia vulgaris & Helminth* & Europe & Tannins & Guarrera (1999) \\
\hline Macleaya cordata & Nematode & Asia & Isoquinoline alkaloids & Satou et al. (2002) \\
\hline Paeonia suffriticosa & Nematode & Asia & $\begin{array}{l}\text { Gallotannins, } \\
\text { condensed tannins }\end{array}$ & Mohamed et al. (2000) \\
\hline Mallotus philippinensis & Cestode & South-eastern Asia & Glycosides & Akhtar \& Ahmad (1992) \\
\hline Sesbania sesban & Nematode & $\begin{array}{c}\text { South-eastern } \\
\text { Asia, Africa }\end{array}$ & Condensed tannins & Ibrahim (1992) \\
\hline Balanites aegyptiana & Nematode & Africa & Saponins & Ibrahim (1992) \\
\hline Chenopodium ambrosioides & Nematode & Central America & Ascaridol & Guarrera (1999) \\
\hline
\end{tabular}

*The parasite class was not identified in these studies.

PSM on the development of parasite larvae, their mobility and their ability to feed. For example, condensed tannin extracts reduce the migration of third-stage larvae of Trichostrongylus colubriformis, a sheep nematode of economic importance in temperate climates (Lorimer et al. 1996). This finding has been taken as evidence of the anthelmintic effects of this extract. Similarly, in vitro use of a commercially-available condensed tannin plant extract causes reduced survival and development of sheep and rat nematode larvae (Athanasiadou et al. 2001; Butter et al. 2001). Other in vitro studies have shown that both purified condensed tannins and terpenoids from several legumes reduce the mobility and consequent migration ability of ovine nematode larvae (Molan et al. 2000, 2003). However, in vitro testing of the antiparasitic properties of PSM can be criticised as being inappropriate, for two main reasons. First, the majority of in vitro assays have been developed for completely different purposes, i.e. the identification of anthelmintic-resistant strains of nematodes and to indicate the efficacy of chemically-synthesised anthelmintic compounds. Although strong correlation has been reported between in vitro assays and in vivo evidence for anthelmintic efficacy and drug resistance, it is not yet clear whether a similar relationship would also be applicable for PSM testing. Second, in vitro assays are mainly performed with the free-living stages rather than the parasitic stages of nematodes, because the former are easier to obtain and the parasitic stages of nematodes cannot be maintained alive outside their host for $>24 \mathrm{~h}$, which is often an inadequate period for in vitro testing. Thus, it is often questionable whether the in vitro assays with pre-parasitic stages are relevant to in vivo conditions.

There is some further justification for this criticism, because it is often difficult to reproduce by in vivo experimentation the dose-related evidence of the antiparasitic activity of PSM clearly demonstrated in vitro. The apparent conflict of evidence may be partly related to a difference in the PSM concentrations used in vitro and those achieved in vivo. In general, there are two main issues that arise during in vitro testing, i.e. the PSM to use and the concentrations to test it at. A source of PSM is chosen usually on the basis of evidence in the literature. Crude or purified extracts from the plant are then used for in vitro testing. This procedure may not give results that are always relevant to the in vivo situation. An example is related to the possible antiparasitic properties of the condensed tannins contained in chicory (Cichorium intybus). Purified condensed tannins from chicory have shown in vitro inhibitory activity against deer nematodes (Molan et al. 2003). However, the condensed tannin content of chicory, at approximately $0.5 \mathrm{~g} / \mathrm{kg} \mathrm{DM}$ is very low, similar to that of grass and clover (Barry, 1998). As a consequence, it is unlikely that the concentrations that have been shown to be successful in vitro can actually be achieved in vivo. Thus, although this example demonstrates the antiparasitic activity of the condensed tannins present in chicory, it is highly unlikely that the antiparasitic effects of chicory observed in vivo (Hoskin et al. 1999) are actually a result of the condensed tannins it contains.

A new methodology for testing the antiparasitic properties of plants in vitro has recently been developed. In contrast to the approach described earlier, there is no need for the initial identification either of the active PSM or of the concentrations to be tested. For example, the inhibitory activity of forages rich in PSM on the feeding and migration ability of an intestinal parasite has been tested in vitro by using rumen fluid taken from sheep grazing pure stands of PSM-rich plants (S Athanasiadou, O Tzamaloukas, I Kyriazakis, F Jackson and RL Coop, unpublished results). This material has been used in order to relate the PSM concentrations utilised in vitro to those observed in vivo. It is appreciated that the use of rumen material for PSM testing may also have its disadvantages. For example, the possible lack of PSM activity in the rumen material does not necessarily mean lack of activity in general, as PSM might become active in the lower parts of the gastrointestinal tract (see p. 634). Despite this limitation, this method seems to have more advantages than the approach described earlier.

The apparent conflict between the evidence from in vitro and in vivo studies could be a result of the difference in the conditions prevailing in the in vitro assays compared with those present in the gastrointestinal tract of grazing herbivores. It could be the case that as a result of the conditions present in the gastrointestinal tract a PSM that has activity in vitro could be inactive in vivo. An example is a condensed tannin extract that shows strong antiparasitic activity against abomasal nematodes of sheep 
in vitro (Athanasiadou et al. 2001), but when this extract is tested in vivo no effect on the abomasal parasite burden of parasitised animals is observed (Athanasiadou et al. 2001). This difference could have arisen because of the difference in the conditions in vitro and in vivo. Condensed tannins are polyphenolic compounds with a high affinity for proteins (Mueller-Harvey \& McAllan, 1992). The majority of condensed tannins present in the abomasum of sheep fed on tannin-rich forages have been found in complexes with protein and are thus probably unavailable for action against parasites (Terrill et al. 1994). In the small intestine, on the other hand, the complexes formed between condensed tannins and protein are expected to break down and free the tannins for action against parasites (Hagerman, 1989; Hagerman \& Butler, 1991). Support for this explanation is provided by good agreement between the in vitro and in vivo assessments of the antiparasitic activity of condensed tannins against intestinal nematodes (Athanasiadou et al. 2001). It is likely, therefore, that under certain circumstances parasitised animals might not be able to experience the beneficial effects of anthelmintic compounds observed in vitro (Fig. 1). In addition, the habitat niche occupied by the parasite in the gastrointestinal tract may reduce the contact between parasite and the PSM. In in vitro studies the parasite will always be in direct contact with the PSM. Lack of contact could account for the absence of activity of PSM in vivo against certain stages of Teladorsagia circumcincta, an abomasal nematode of sheep that usually parasitises the glands rather than the lumen of the abomasum (Urquart et al. 1996).

In conclusion, in vitro tests can provide a valuable means for large-scale plant screening, when in vivo screening would be unrealistic because of time and resource constraints. It is, however, important that in vitro results should always be validated in vivo before making generalised and firm conclusions on the antiparasitic properties of PSM.

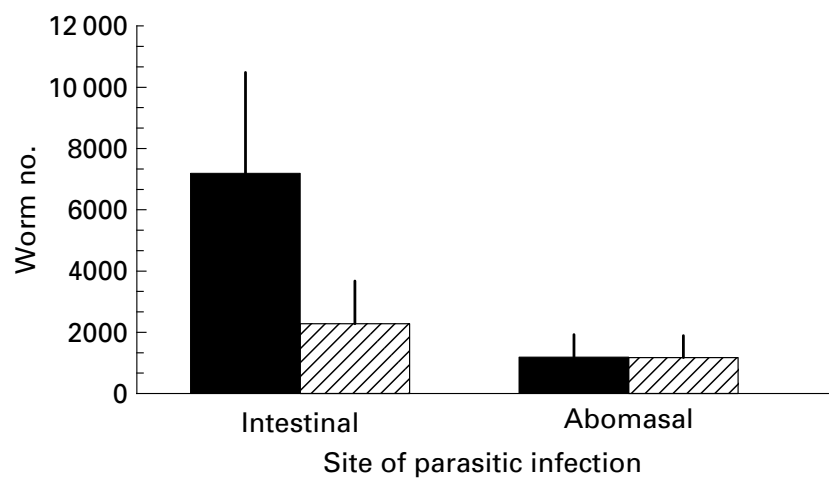

Fig. 1. Total worm population recovered in sheep infected with either an intestinal (Trichostrongylus colubriformis) or an abomasal (Haemonchus contortus) nematode species on day 1 of experiment. On day 28 sheep were drenched with $80 \mathrm{~g}$ commercially-available condensed tannin extract (Quebracho extract)/kg food intake ( $\square$ ). Undrenched controls are also shown $(\boldsymbol{\square})$. The values are backtransformed means and $95 \% \mathrm{Cl}$ represented by vertical bars. (From Athanasiadou et al. 2001.)

\section{The failure of in vivo studies to be consistent}

Two methods have been used to investigate the antiparasitic effects of PSM in vivo. The first method involves indoor supplementation studies. Animals with no previous experience of parasites are infected with a given number of a specific parasite species. They then receive either a PSM supplement or no supplement. In these conditions, in which the level of the parasitic infection, the immune and nutritional state of the animals and particularly the PSM amount consumed are all controlled, the evidence for the antiparasitic properties of PSM is strong and abundant. For example, supplementation of parasitised goats with an extract from Mallotus philippinensis, which is rich in glycosides, results in an $80 \%$ reduction in the mixednematode parasitic burden compared with unsupplemented controls (Akhtar \& Ahmad, 1992). Sheep parasitised with an intestinal nematode and given access to a tannin-rich food show a 50\% lower nematode egg excretion and parasite worm burden than sheep offered a tannin-free food (Athanasiadou et al. 2000). In some cases the antiparasitic efficacy of PSM-rich extracts is similar to that of broadspectrum anthelmintic drugs; Satrija et al. (1994) almost eliminated intestinal nematodes within $7 \mathrm{~d}$ of supplementation with PSM.

The second method used to investigate the antiparasitic effects of PSM involves grazing the animals on PSM-rich forages, which is more relevant to normal agricultural practice. It is the conflicting evidence from these studies that casts major doubt on the antiparasitic effects of PSM. For example, sheep grazing on birdsfoot trefoil (Lotus spp.; a forage that is rich in condensed tannins) have been shown to reduce their level of parasitism in some studies (Marley et al. 2003), but not in others (Niezen et al. $1998 a, b)$. Similar inconsistency has been reported when sheep graze sulla (Hedysarium coronarium), another tanninrich forage (Niezen et al. 1995, 2002). One of the reasons for the conflicting evidence from grazing studies might be the variable PSM content of the forages. Both PSM concentration and structure can vary between experimental sites and years, and even within the same grazing period (Barry \& MacNabb, 1999). These variations occur because PSM production depends on many factors, such as the extent of herbivory, weather conditions and pathogen infestation (Hagerman \& Butler, 1991). As the antiparasitic properties of PSM are likely to be related to their structure and content in the plants, the large variability in the antiparasitic effects of PSM observed in grazing studies is likely to be directly related to this variation. The control over the amount of PSM ingested by parasitised herbivores in supplementation studies may result in reduced variability in the antiparasitic activity of PSM and a consequently smaller extent of inconsistency than that seen in grazing studies.

In addition to the variation in PSM content and structure, the evidence from different grazing studies might be seen to be in conflict for artificial reasons, as it may be the outcome of inappropriate comparisons. In grazing studies the levels of parasitism are measured in animals grazing on forages rich in PSM and in controls. The forages may also differ in characteristics other than 
their PSM contents. When sheep are grazed on birdsfoot trefoil (expected to have 40-100 g condensed tannins $/ \mathrm{kg}$ DM according to Barry \& Duncan, 1984) and grass with traces of condensed tannins (Niezen et al. 1998b), the sheep on birdsfoot trefoil show a lower level of parasitism (a lower number of nematode eggs excreted in the faeces) than sheep on grass. As the crude protein content of the birdsfoot trefoil would be expected to be approximately twice that of the grass (Barry \& Duncan, 1984), this difference could contribute to the difference in parasitism (Coop \& Kyriazakis, 1999). In addition, the difference could be a result of the difference in the levels of condensed tannins or the protein content, or neither of these factors. There are other examples in the literature that may be open to similar interpretation (Niezen et al. 1993, 1994; Robertson et al. 1995).

The view that the beneficial effects of birdsfoot trefoil in the study of Niezen et al. (1998b) are a result of its nutritional effects is supported by the work of Niezen et al. (1998a). They reported that sheep grazing on birdsfoot trefoil show a similar level of parasitism to those grazing on lucerne (Medicago sativa; a forage low in tannins). In this case parasitised sheep grazing on birdsfoot trefoil are not expected to have any nutritional advantages compared with those grazing on lucerne, as the protein intake is expected to be similar in both cases. Providing that the content and structure of the condensed tannins in birdsfoot trefoil is similar in both studies, then the lack of a reduction in parasitism in the Niezen et al. (1998a) study supports the view that previously reported beneficial effects of birdsfoot trefoil (Niezen et al. 1998b) may have been a result of the nutritional benefits of birdsfoot trefoil rather than the antiparasitic effects of condensed tannins contained in it. In grazing studies it is difficult to draw definite conclusions on the potential antiparasitic properties of PSM, as the contents of other nutrients as well as that of PSM may be variable. Thus, caution is required when comparisons are made between PSM-rich and control forages. The great advantage of supplementation studies is that parasitised animals are given access to foods that can be nutritionally identical, with the exception of their PSM contents. Thus, it is probably more appropriate to draw conclusions on the antiparasitic properties of PSM from supplementation studies rather than from grazing studies.

It is strongly recommended that comparisons across grazing and in vitro studies should be made with caution, as they might not always provide reliable information. It would be more appropriate and relevant to relate the antiparasitic properties of PSM to their structure and content across PSM-rich forages and studies. In other words, it would be more informative to suggest that condensed tannins at the $\mathrm{x}$ level and $\mathrm{y}$ structure contained in birdsfoot trefoil have antiparasitic properties rather than that birdsfoot trefoil has antiparasitic properties. Unfortunately, such essential information is usually absent from the description of PSM-rich forages used in grazing experiments, and thus comparisons are presently made at the level of the forage, rather than the PSM content and chemical structure.

Supplementation studies in which PSM consumption and experimental conditions can be managed have provided convincing evidence of the antiparasitic action of certain PSM. In vitro evidence provides further support that PSM such as condensed tannins, alkaloids and glycosides have the ability to exert antiparasitic properties. However, despite their potential positive effects, PSM consumption does not necessarily benefit parasitised herbivores, as their excessive consumption can result in detrimental effects related to their anti-nutritional properties. In the next part of the paper the conditions under which parasitised animals may be able to benefit from PSM consumption will be explored by taking into account both the positive and negative effects of PSM.

\section{Positive and negative consequences of plant secondary metabolite consumption}

The antiparasitic activity of PSM could be seen as an alternative way of controlling parasitic infections in agricultural systems. However, in addition to their antiparasitic properties, PSM are better known for their anti-nutritional properties. The consumption of PSM-rich plants by herbivores can result in reduced intake, weight loss, toxicity and death (Milgate \& Roberts, 1995; Waghorn \& McNabb, 2003). For example, condensed tannin consumption has been shown to be associated with reduced food intake, reduced digestibility and impaired rumen metabolism (Barry \& McNabb, 1999; Min et al. 2003). Some types of condensed tannins are also responsible for mucosal toxicity and consequently a reduction in nutrient absorption (Reed, 1995; Dawson et al. 1999). Saponins have also been considered responsible for reduced food intake and growth impairment (Applebaum \& Birk, 1979; Milgate \& Roberts, 1995). They have also been associated with haemolytic action and bloat in ruminants. Excessive consumption of alkaloids, glycosides and terpenoids can result in lesions in the nervous system (Conn, 1979; Mabry \& Gill, 1979). It is thus evident that in order for PSM to have any role in parasite control it is necessary to ensure that parasitised herbivores can obtain a net benefit from PSM ingestion. For this reason both positive (antiparasitic) and negative (anti-nutritional) effects of PSM consumption should be evaluated based on the same measures. The common measure that can be used for this purpose is the performance of parasitised hosts, because it is expected that both the level of parasitism and PSM consumption will affect host performance.

As a consequence of the antiparasitic effects of PSM, the performance of parasitised herbivores is expected to improve following the consumption of PSM (Coop et al. 1982; Sykes, 1994). Thus, the greater the reduction in the level of parasitism, the larger should be the improvement in the performance of parasitised herbivores. Many parasitological studies have taken this 'pharmacological' approach to evaluate the antiparasitic properties of PSM. The main aim of PSM administration has been the achievement of effective parasite control, without taking into account the potential detrimental effects of PSM consumption on host performance (Akhtar \& Ahmad, 1992; Ketzis et al. 2002; Satrija et al. 1994). However, the consumption of PSM can also impair herbivore performance as a result of their anti-nutritional properties. Thus, 
Table 3. The consequences of plant secondary metabolite (PSM) consumption on the performance of parasitised herbivores in relation to the performance of non-parasitised herbivores not given access to $\mathrm{PSM}^{*}$

\begin{tabular}{|c|c|c|c|}
\hline \multirow{2}{*}{$\begin{array}{l}\text { Performance of } \\
\text { non-parasitised } \\
\text { herbivores (NPH; } \\
\text { arbitrary units) }\end{array}$} & \multicolumn{2}{|c|}{$\begin{array}{l}\text { Performance of parasitised herbivores } \\
\qquad(\mathrm{PH})\end{array}$} & \multirow{2}{*}{$\begin{array}{c}\text { Consequences of PSM } \\
\text { consumption on parasitised } \\
\text { herbivores }\end{array}$} \\
\hline & $\begin{array}{l}-\mathrm{PSM} \\
(\% \mathrm{NPH})\end{array}$ & $\begin{array}{l}+\mathrm{PSM} \\
(\% \mathrm{NPH})\end{array}$ & \\
\hline 100 & 80 & 60 & Net cost $(A)$ \\
\hline 100 & 80 & 80 & Neither cost nor benefit (B) \\
\hline 100 & 80 & $81-100$ & Net benefit $(\mathrm{C})$ \\
\hline
\end{tabular}

(A), When the performance of herbivores given is lower than that from their PSM-free counterparts, a net cost is expected from PSM consumption; (B), when the performance of PSM and PSM-free herbivores is similar, then parasitised herbivores obtain neither cost nor benefit from PSM consumption; (C), when the performance of PSM herbivores is improved compared with that of PSM-free herbivores, then the parasitised herbivores obtain a net benefit from PSM consumption.

*It is assumed that consumption of PSM will lead to a reduction in parasitism.

PSM can have a role in parasite control only if parasitised herbivores can obtain a net benefit on their performance from PSM ingestion. This net benefit can only be achieved if the antiparasitic (positive) effects of PSM outweigh the anti-nutritional (negative) consequences on host performance.

Whether the antiparasitic effects of PSM can outweigh their anti-nutritional consequences in relation to the performance of the parasitised host depends on the strength of the effects of PSM. Table 3 shows the three possible consequences of PSM consumption by parasitised herbivores. First, if the cost from the anti-nutritional activity of PSM on the performance of parasitised herbivores is greater than the benefit from its antiparasitic activity, then parasitised herbivores are expected to show a net cost, i.e. a reduction in performance, from the PSM consumption (Table 3; case A). For example, two recent studies (Athanasiadou et al. 2000; Butter et al. 2000) have reported that sheep parasitised with $T$. colubriformis have a lower body-weight gain than their non-parasitised counterparts. Both studies have also shown that parasitised sheep given access to a food that contains condensed tannins reduce their level of parasitism, but also reduce their final body weight by $20 \%$ when compared with parasitised sheep offered access to a tannin-free food. Similarly, the administration of a condensed tannin extract at $80 \mathrm{~g} / \mathrm{kg}$ food intake to sheep infected with the same nematode results in a reduced level of parasitism, but also in penalties on the performance of sheep when compared with their PSM-free infected counterparts (Athanasiadou et al. 2001). However, when the same condensed tannin extract is administered at $40 \mathrm{~g}$ per $\mathrm{kg}$ food intake, the level of parasitism is reduced, but the performance of these sheep is not impaired. Thus, in the latter case parasitised sheep obtain neither a benefit nor a cost from PSM consumption in terms of their performance (Table 3; case B). This lack of a net effect on host performance is probably attributable to the fact that the anti-nutritional effects of PSM offset a potential benefit on host performance mediated through the reduced level of parasitism.

On the other hand, if the antiparasitic activity of PSM has greater consequences on the performance of parasitised hosts than its anti-nutritional activity, then parasitised herbivores should be able to obtain a net benefit, i.e. an improved performance, from the consumption of PSM
(Table 3; case C). Despite a diligent search of the literature, no examples of supplementation studies have been found to support the latter possibility. In supplementation studies in which performance measurements have been recorded it seems that the performance of parasitised herbivores is either impaired or remains unaltered compared with unsupplemented controls. It is unfortunate that the majority of supplementation studies that have most robustly demonstrated the antiparasitic effects of PSM do not also report the effects of PSM on host performance. Thus, it is not possible to draw useful conclusions on the potential use of PSM for parasite control in ruminants.

In contrast to supplementation studies, there is a large body of evidence suggesting that grazing on PSM-rich forages can result in a reduced level of parasitism and improved performance of parasitised hosts (Niezen et al. 1995, 1998b; Hoskin et al. 1999; Marley et al. 2003). Although parasitised hosts have undoubtedly obtained a benefit following the consumption of PSM-rich forages in the studies mentioned earlier, it is not always clear how this benefit has been achieved. It has already been mentioned that PSM-rich forages are usually leguminous and therefore nutritionally advantageous compared with conventional grazing forages. Consequently, the improved performance of parasitised hosts grazing on such forages could be attributable to either the nutritional benefits or the antiparasitic activity of the PSM-rich forages. The majority of the grazing studies have not been designed to distinguish between the different effects of the PSM-rich forages. Awareness on how such beneficial effects have been achieved would help to improve the understanding of the consequences of PSM consumption and determine the appropriate grazing strategies for incorporating PSM-rich forages for parasite control. The differentiation between the nutritional and antiparasitic effects of PSM-rich forages could be facilitated by the inclusion of parasitised and non-parasitised animals in the same studies. Any differences in the performance of non-parasitised animals grazing on PSM-rich and control forages would provide evidence of the nutritional effects of PSM-rich forages. In addition, as increased nutrient supply during the course of a parasitic infection has been shown to enhance immunity to parasites (Coop \& Kyriazakis, 1999; Houdijk et al. 2001), short experimental studies that minimise the interactions between nutrition and immunity would also 
assist in distinguishing the antiparasitic from the nutritional effects of PSM-rich forages (Athanasiadou et al. 2004; Tzamaloukas et al. 2004).

\section{A role for plant secondary metabolite-rich forages in parasite control: future research directions}

The role for PSM-rich forages in parasite control could be considered as part of either a short-term or a long-term strategy. In the first case parasitised animals would be provided with PSM-rich forages for short periods of time, in order to reduce their level of parasitism, and then they would return to conventional grazing. This option, in which PSM-rich forages would act as 'de-worming' paddocks, would be extremely attractive if they are effective, as potential adverse effects of PSM consumption on the performance of parasitised hosts would only be for the short term. Indeed, if short-term PSM consumption could reduce the level of parasitism, as has already been the case for certain PSM (Paolini et al. 2003; Min et al. 2004; Tzamaloukas et al. 2004), then potential short-term penalties on animal performance could actually lead to long-term benefits. These long-term benefits would be translated as a lower level of parasitism in the grazing environment, thus lower parasite exposure and greater protection from parasites in subsequent grazing seasons. In addition to animals being transferred to 'de-worming' paddocks for parasite treatment, livestock could also be given the choice of grazing PSM-rich forages whenever they wished. There is already evidence suggesting that non-parasitised grazing animals can distinguish between and avoid plants rich in PSM, probably because of their anti-nutritional properties (Provenza et al. 1990; Villalba $\&$ Provenza, 2002). However, although no evidence is yet available to indicate whether parasitised domestic herbivores can select a diet rich in PSM in order to combat their parasitic infection (Hutchings et al. 2003), this approach remains an attractive possibility (Huffman, 2003). Evidence indicating the ability of animals to select a diet to combat parasitism, i.e. to self medicate, will facilitate the design of sustainable and efficient grazing systems for ruminant production that provide for parasite control.

Second, PSM consumption could also be considered as part of a long-term strategy in which parasitised animals would be given access to PSM-rich forages for most of the grazing season. Long-term grazing on PSM-rich forages could be considered advantageous in cases where shortterm PSM consumption is not effective against parasites. However, in order for a long-term approach to be sustainable and beneficial for parasitised animals, the costs of long-term PSM consumption would need to be thoroughly determined. Long-term consequences of PSM consumption have been reported to be more severe than the short-term ones. For example, the consumption of certain PSM for periods longer than a few days seems to be associated with irreversible negative post-ingestive consequences (Reed, 1995; Silanikove et al. 2001). In the short term such negative consequences would have been reversible. Such problems arising from the long-term exposure to PSM may be overcome by offering sequentially a combination of PSM-rich forages to parasitised animals for short periods of time. The combination of more than one PSM-rich forage could minimise penalties to host performance by dilution of the anti-nutritional effects of PSM and could offer even better parasite control through synergistic antiparasitic effects of PSM (e.g. through the effects of different PSM on different parasite species).

To date, ethnoveterinary and 'pharmacological' evidence of the antiparasitic properties of PSM have focused on the medicinal effects of PSM. The findings have led to the identification of active compounds and the synthesis of antiparasitic drugs, such as santonine and ivermectine (Clark, 1996). However, both sources of evidence have so far failed to report and take into account possible detrimental effects of PSM consumption on the performance of parasitised herbivores, attributed to their antinutritional properties. In the present paper the need for a holistic approach to the antiparasitic effects of PSM through simultaneous evaluation of the costs and benefits of PSM consumption has been emphasised. This knowledge is important for the incorporation of PSM-rich forages into grazing systems for parasite control. It should be noted, however, that even with this evaluation it is unlikely that complete parasite control can be achieved through the use of PSM-rich forages alone. No evidence available to date suggests complete parasite control as a result of PSM consumption similar to that achieved by the use of antiparasitic drugs, i.e. $100 \%$ reduction in parasites without adverse effects on animal performance. As a consequence, the development of non-chemical sustainable parasite control strategies will require a multi-angle approach, in which strategic use of bioactive forages is combined with nutritional control, genetic selection for increased resistance, vaccination, biological control and, whenever inevitable, the use of drugs to combat gastrointestinal parasitism.

\section{Acknowledgement}

We would like to thank our colleagues Gerry Emmans, Bert Tolkamp and Jos Houdijk for their valuable comments and criticism of previous drafts of this paper. Part of the work presented here has been performed in collaboration with our colleagues from the Parasitology Division at the Moredun Research Institute. This work was supported by the European Commision, project QLRT-2000-01843, as part of a collaborative programme between Scotland, France, Spain, Sweden and The Netherlands. The Scottish Executive Environment and Rural Affairs Department has financially supported part of the work presented.

\section{References}

Akhtar MS \& Ahmad I (1992) Comparative efficacy of Mallotus philippinensis fruit (Kamala) or Nilzan drug against gastrointestinal cestodes in Beetal goats. Small Ruminants Research 8, 121-128.

Applebaum SW \& Birk Y (1979) Saponins. In Herbivores. Their Interactions with Secondary Plant Metabolites, pp. 539-566 [GA Rosental and TH Janzen, editors]. London: Academic Press. 
Athanasiadou S, Kyriazakis I, Jackson F \& Coop RL (2000) Consequences of long-term feeding with condensed tannins on sheep parasitised with Trichostrongylus colubriformis. International Journal for Parasitology 30, 1025-1033.

Athanasiadou S, Kyriazakis I, Jackson F \& Coop RL (2001) Direct anthelmintic effects of condensed tannins towards different gastrointestinal nematodes of sheep: in vitro and in vivo studies. Veterinary Parasitology 99, 205-219.

Athanasiadou S, Tzamaloukas O, Kyriazakis I, Jackson F \& Coop RL (2004) Direct effects of bioactive forages in sheep infected with Trichostrongylus colubriformis. Proceedings of the British Society of Animal Science, 52.

Barry TN (1998) The feeding value of chicory (Cichorium intybus) for ruminant livestock. Journal of Agricultural Science, Cambridge 131, 251-257.

Barry TN \& Duncan SJ (1984) The role of condensed tannins in the nutritional value of Lotus pedunculatus 1 . Voluntary intake. British Journal of Nutrition 51, 485-491.

Barry TN \& McNabb WC (1999) The implications of condensed tannins on the nutritive value of temperate forages fed to ruminants. British Journal of Nutrition 81, 263-272.

Bernays EA, Driver GC \& Bilgener M (1989) Herbivores and plant tannins. Advances in Ecological Research 19, 263-302.

Butter NL, Dawson JM, Wakelin D \& Buttery PJ (2000) Effect of dietary tannin and protein concentration on nematode infection (Trichostrongylus colubriformis) in lambs. Journal of Agricultural Science, Cambridge 134, 89-99.

Butter NL, Dawson JM, Wakelin D \& Buttery PJ (2001) Effect of dietary condensed tannins on gastrointestinal nematodes. Journal of Agricultural Science, Cambridge 137, 461-469.

Clark AM (1996) Natural products as a resource for new drugs. Pharmaceutical Research 13, 1133-1141.

Conn EE (1979) Cyanide and cyanogenic glycosides. In Herbivores. Their Interactions with Secondary Plant Metabolites, pp. 387-412 [GA Rosental and TH Janzen, editors]. London: Academic Press.

Coop RL \& Kyriazakis I (1999) Nutrition-parasite interaction. Veterinary Parasitology 84, 187-204.

Coop RL \& Kyriazakis I (2001) Influence of host nutrition on the development and consequences of nematode parasitism in livestock. Trends in Parasitology 17, 325-330.

Coop RL, Sykes AR \& Angus KW (1982) The effect of three levels of intake of Ostertagia circumcincta larvae on growth rate, food intake and body composition of growing lambs. Journal of Agricultural Science, Cambridge 98, 247-255.

Dawson JM, Buttery PJ, Jenkins D, Wood CD \& Gill M (1999) Effects of dietary Quebracho tannin on nutrient utilisation and tissue metabolism in sheep and rats. Journal of the Science of Food and Agriculture 79, 1423-1430.

Githiori JB, Hoglund J, Waller PJ \& Baker RL (2002) Anthelmintic activity of preparations derived from Myrsine africana and Rapanea melanophloeos against the nematode parasite, Haemonchus contortus of sheep. Journal of Ethnopharmacology 80, 187-191.

Gray GD (1997) The use of genetically resistant sheep to control nematode parasitism. Veterinary Parasitology 72, 345-366.

Guarrera PM (1999) Traditional antihelmintic, antiparasitic and repellent uses of plants in Central Italy. Journal of Ethnopharmacology 68, 183-192.

Hagerman AE (1989) Chemistry of tannin-protein complexation. In Chemistry and Significance of Condensed Tannins, pp. 323-333 [RW Hemingway and JJ Karchesy, editors]. New York and London: Plenum Press.

Hagerman AE \& Butler LG (1991) Tannins and lignins. In Herbivores: Their Interaction with Secondary Plant Metabolites, pp. 355-376 [GA Rosental and MR Berenbaum, editors]. San Diego, CA: Academic Press.
Harborne JB (1999) An overview of antinutritional factors in higher plants. In Secondary Plant Products. Antinutritional and Beneficial Actions in Animal Feeding, pp. 7-16 [JC Caygill and I Mueller-Harvey, editors]. Nottingham: Nottingham University Press.

Hoskin SO, Barry TN, Wilson PR, Charleston WAG \& Hodgson J (1999) Effects of reducing anthelmintic input upon growth and faecal egg and larval counts in young farmed deer grazing chicory (Chicorium intybus) and perennial ryegrass (Lolium perenne)/white clover (Trifolium repens) pasture. Journal of Agricultural Science, Cambridge 132, 335-345.

Houdijk JGM \& Athanasiadou S (2003) Direct and indirect effects of host nutrition on ruminant gastrointestinal nematodes. In Matching Herbivore Nutrition to Ecosystems Biodiversity, pp. 213-236 [Lt' Mannetje, L Ramirez-Aviles, Sandoval-Castro and JC Ku-Vera, editors]. Yucatan, Mexico: Universita Autonoma de Yucatan.

Houdijk JGM, Jessop NS \& Kyriazakis I (2001) Nutrient partitioning between reproductive and immune functions in animals. Proceedings of the Nutrition Society 60, 515-525.

Huffman MA (2003) Animal self-medication and ethno-medicine: exploration and exploitation of the medicinal properties of plants. Proceedings of the Nutrition Society 62, 371-381.

Hutchings MR, Athanasiadou S, Kyriazakis I \& Gordon IJ (2003) Can animals use foraging behaviour to combat parasites? Proceedings of the Nutrition Society 62, 361-370.

Ibrahim AM (1992) Anthelmintic activity of some Sudanese anthelmintic plants. Phytotherapy Research 6, 155-157.

International Institute of Rural Reconstruction (1994) Ethnoveterinary Medicine in Asia. An Information Kit on Traditional Animal Health and Care Practices, vol. 2, Ruminants. Silang, Cavite, The Phillippines: International Institute of Rural Reconstruction

Jackson F \& Coop RL (2000) The development of anthelmintic resistance in sheep nematodes. Parasitology 120, Suppl., S95-S107.

Julien J, Gasquet M, Maillard C, Balansard G \& Timon-David P (1985) Extracts of the ivy plant, Hedera helix, and their anthelminthic activity on liver flukes. Plant Medica 3, 205-208.

Karban R, Agrawal AA, Thaler JS \& Adler LS (1999) Induced plant responses and information content about risk of herbivory. Trends in Ecology and Evolution 14, 443-447.

Ketzis JK, Taylor A, Bowman DD, Brown DL, Warnick LD \& Erb HN (2002) Chenopodium ambrosioides and its essential oil as treatments for Haemonchus contortus and mixed adultnematode infections in goats. Small Ruminant Research 44, 193-200.

Larsen M (1999) Biological control of helminths. International Journal for Parasitology 29, 139-146.

Lorimer SD, Perry NB, Foster LM \& Burgess EJ (1996) A nematode larval motility inhibition assay for screening plant extracts and natural products. Journal of Agriculture and Food Chemistry 44, 2842-2845.

Mabry TJ \& Gill JE (1979) Sesquiterpenes lactones and other terpenoids. In Herbivores. Their Interactions with Secondary Plant Metabolites, pp. 501-537 [GA Rosental and TH Janzen, editors]. London: Academic Press.

Marley CL, Cook R, Keatinge R, Barrett J \& Lampkin NH (2003) The effect of birdsfoot trefoil (Lotus corniculatus) and chicory (Cichorium intybus) on parasite intensities and performance of lambs naturally infected with helminth parasites. Veterinary Parasitology 112, 147-155.

Milgate J \& Roberts DCK (1995) The nutritional and biological significance of saponins. Nutrition Research 15, 1223-1249.

Min BR, Barry TN, Attwood GT \& McNabb WC (2003) The effect of condensed tannins on the nutrition and health of 
ruminants fed fresh temperate forages: a review. Animal Feed Science and Technology 106, 3-19.

Min BR, Pomroy WE, Hart SP \& Sahlu T (2004) The effect of short-term consumption of a forage containing condensed tannins on gastro-intestinal nematode parasite infections in grazing wether goats. Small Ruminant Research 51, 279-283.

Mohamed ASA, Mori T, Islam SQ, Sato M \& Yamasaki T (2000) Lethal activity of gallo- and condensed tannins against the free-living soil-inhabiting nematode Caenorhabditis elegans. Journal of Pesticide Science 25, 410-415.

Molan A, Waghorn GC, Min BR \& McNabb WC (2000) The effects of condensed tannins from seven herbages on Trichostrongylus colubriformis larval migration in vitro. Folia Parasitologica 47, 39-44.

Molan AP, Duncan A, Barry TN \& McNabb WC (2003) Effects of condensed tannins and sesquiterpene lactones extracted from chicory on the viability of deer lungworm larvae. Proceedings of the New Zealand Society of Animal Production 60, 26-29.

Mueller-Harvey I \& McAllan AB (1992) Tannins: their biochemistry and nutritional properties. Advances in Plant Cell Biochemistry and Biotechnology 1, 151-217.

Niezen JH, Charleston WAG, Hodgson J \& Waghorn TS (1993) Effect of four grass species on lamb parasitism and growth. Proceedings of the New Zealand Grassland Association 55, 203-206.

Niezen JH, Charleston WAG, Robertson HA, Shelton D, Waghorn GC \& Green R (2002) The effect of feeding sulla (Hedysarum coronarium) or lucerne (Medicago sativa) on lamb parasite burdens and development of immunity to gastrointestinal nematodes. Veterinary Parasitology $\mathbf{1 0 5}$, 229-245.

Niezen JH, Robertson HA, Waghorn GC \& Charleston WAG (1998a) Production, faecal egg counts and worm burdens of ewe lambs which grazed six contrasting forages. Veterinary Parasitology 80, 15-27.

Niezen JH, Waghorn GC \& Charleston WAG (1998b) Establishment and fecundity of Ostertagia circumcincta and Trichostrongylus colubriformis in lambs fed lotus (Lotus pedunculatus) or perennial ryegrass (Lolium perenne). Veterinary Parasitology 78, 13-21.

Niezen JH, Waghorn TS, Charleston WAG \& Waghorn GC (1995) Growth and gastrointestinal nematode parasitism in lambs grazing either lucerne (Medicago sativa) or sulla (Hedysarum coronarium) which contains condensed tannins. Journal of Agricultural Science, Cambridge 125, 281-289.

Niezen JH, Waghorn TS, Raufautk K, Robertson HA \& McFarlane RG (1994) Lamb weight gain and faecal egg count when grazing one of seven herbages and dosed with larvae for six weeks. Proceedings of the New Zealand Society of Animal Production 54, 15-18.

Paolini V, Dorchies P \& Hoste H (2003) Effects of sainfoin hay on gastrointestinal nematode infections in goats. Veterinary Record 152, 600-601.

Provenza FD, Burritt EA, Clausen TP, Bryant JP, Reichardt PB \& Distel RA (1990) Conditioned flavour aversion: a mechanism for goats to avoid condensed tannins in blackbrush. American Naturalist 136, 810-828.

Reed JD (1995) Nutritional toxicology of tannins and related polyphenols in forage legumes. Journal of Animal Science 73, $1516-1528$.

Robertson HA, Niezen JH, Waghorn GC, Charleston WAG \& Jinlong M (1995) The effect of six herbages on liveweight gain, wool growth and faecal egg count of parasitised ewe lambs. Proceedings of the New Zealand Society of Animal Production 55, 199-201.

Satou T, Akao N, Matsuhashi R, Koike K, Fujita K \& Nikaido T (2002) Inhibitory effect of isoquinoline alkaloids on movement of second-stage larvae of Toxocara canis. Biological \& Pharmaceutical Bulletin 25, 1651-1654.

Satrija F, Nansen P, Bjorn H, Murtini S \& He S (1994) Effects of papaya latex against Ascaris suum naturally infected pigs. Journal of Helminthology 68, 343-346.

Silanikove N, Perevolotsky A \& Provenza FD (2001) Use of tannin-binding chemicals to assay for tannins and their negative post-ingestive effects in ruminants. Animal Feed Science and Technology 91, 69-81.

Smith WD (1999) Prospects for vaccines of helminth parasites of grazing ruminants. International Journal for Parasitology 29, $17-24$.

Sykes AR (1994) Parasitism and production in farm animals. Animal Production 59, 155-172.

Terrill TH, Waghorn GC, Wooley DJ, McNabb WC \& Barry TN (1994) Assay and digestion of ${ }^{14} \mathrm{C}$-labelled condensed tannins in the gastrointestinal tract of sheep. British Journal of Nutrition 72, 467-477.

Tzamaloukas O, Athanasiadou S, Kyriazakis I, Jackson F \& Coop RL (2004) Effects of short-term grazing on bioactive forages on lambs artificially infected with Teladorsagia circumicncta. Proceedings of the British Society of Animal Science, 6.

Urquart GM, Armour J, Duncan JL, Dunn AM \& Jennings FW (1996) Veterinary Helminthology. In Parasitology, pp. 10-26 [GM Urquart, editor]. Oxford: Blackwell.

Villalba JJ \& Provenza FD (2002) Polyethylene glycol influences selection of foraging location by sheep consuming quebracho tannin. Journal of Animal Science 80, 1846-1851.

Waghorn GC \& McNabb WC (2003) Consequences of plant phenolic compounds for productivity and health of ruminants. Proceedings of the Nutrition Society 62, 383-392. 
https://doi.org/10.1079/PNS2004396 Published online by Cambridge University Press 